\title{
Research Paper: Emotional Processing in Patients With Early- and Late-Onset Temporal Lobe Epilepsy
}

Sepideh Batebi ${ }^{1}$ (D), Behrooz Dolatshahi ${ }^{2^{*}}$ (D), Mojtaba Azimian ${ }^{3}$ (D)

1. Department of Clinical Psychology, Faculty of Medicine, Shahid Beheshti University of Medical Sciences, Tehran, Iran.

2. Department of Clinical Psychology, University of Social Welfare and Rehabilitation Sciences, Tehran, Iran.

3. Department of Clinical Sciences, University of Social Welfare and Rehabilitation Sciences, Tehran, Iran.

\begin{tabular}{|c|c|}
\hline \multicolumn{2}{|l|}{$\begin{array}{l}\text { Use your device to scan } \\
\text { and read the article online }\end{array}$} \\
\hline 口象回 & CFtation: Batebi, S., Dolatshahi, B., \& Azimian, M. (2020). Emotional Processing in Patients With Early- and Late-Onset \\
\hline 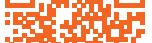 & Temporal Lobe Epilepsy. Journal of Practice in Clinical Psychology, 8(1), 47-56. https://doi.org/10.32598/jpcp.8.1.49 \\
\hline actes & doil'https://doi.org/10.32598/jpcp.8.1.49 \\
\hline
\end{tabular}

(c) (1) (3)

Article info:

Received: 10 Apr 2019

Accepted: 25 Oct 2019

Available Online: 01 Jan 2020

Keywords:

Epilepsy, Temporal lobe epilepsy, Emotions, EarlyOnset, Late-Onset

\section{ABSTRACT}

Objective: Temporal Lobe Epilepsy (TLE) could cause various emotional symptoms due to the damages imposed on the temporal lobe. This study aimed at comparing emotional processing between patients with early- and late-onset TLE and a healthy group.

Methods: In a cross-sectional study, after a definitive diagnosis of TLE, 60 patients were compared with 60 healthy controls without any epilepsy to identify emotional-processing styles in them. The research instruments were the Emotional Processing Scale and the Diagnostic and Statistical Manual of Mental Disorders, $5^{\text {th }}$ Edition (DSM-5)-based psychiatric interview. The obtained data were analyzed using a multivariate analysis of variance by SPSS.

Results: The present study results suggested significant differences in emotional processing between the investigated epileptic and non-epileptic groups $(\mathrm{P}<0.01)$. Besides, emotional processing indicated significant differences in intrusion, the lack of attunement, and dissociation subscales between patients with early-onset TLE, compared to those with late-onset TLE.

Conclusion: The obtained data revealed more emotional processing dysfunction in patients affected by TLE, compared to the non-epileptics. Besides, in some subscales of emotional processing, there was more malfunctioning in the early-onset TLE group, compared to the late-onset TLE group.

\section{* Corresponding Author:}

Behrooz Dolatshahi, $P h D$

Address: Department of Clinical Psychology, University of Social Welfare and Rehabilitation Sciences, Tehran, Iran

Tel: +98 (21) 22180045

E-mail:dolatshahee@yahoo.com 


\section{Highlights}

- TLE could cause various emotional symptoms due to the damages imposed on the temporal lobe.

- There are sigificace differences in emotional processing between the investigated epileptic and non-epileptic groups.

- There are more emotional processing dysfunction in patients affected by TLE, compared to the non-epileptics.

\section{Plain Language Summary}

Temporal Lobe Epilepsy (TLE) could cause various emotional symptoms due to the damages imposed on the temporal lobe. Some patients with epilepsy were compared to patients with no epilepsy to identify emotional-processing styles in them. The obtained data were analyzed using a multivariate analysis of variance by SPSS. The results suggested significant differences in emotional processing between the investigated epileptic and non-epileptic groups. Also, emotional processing indicated significant differences in intrusion, the lack of attunement, and dissociation subscales between patients with early-onset TLE, compared to those with late-onset TLE. The data revealed more emotional processing dysfunction in patients affected by TLE, compared to the non-epileptics. This study aimed to compare emotional processing between patients with early- and late-onset TLE and a healthy group.

\section{Introduction}

pilepsy is a prevalent neurological disease, which imposes a heavy burden on the affected families and the society, including psychological and economic problems (Elliott \& Richardson, 2014; Baulac et al., 2015). In patients with Temporal

Lobe Epilepsy (TLE), Mesial Temporal Sclerosis (MTS), the loss of neurons and gliosis around the hippocampus, entorhinal, and amygdala complexes have been reported (Cukiert, Cukiert, Burattini, \& Lima, 2014; Kang et al., 2016). The amygdala damage separately occurs in patients with TLE (Lv et al., 2014; Yilmazer-Hanke, O’Loughlin, \& McDermott, 2016).

Neuropsychological studies and neuroimaging have supported the effectiveness of the amygdala in cognitive and emotional processing, especially, in negative emotions; consequently, the damages to amygdala lead to deficits in emotional evaluation and processing (Dellacherie, Hasboun, Baulac, Belin, \& Samson, 2011; Schmidt et al., 2014; Mier et al., 2014; Monti, \& Meletti, 2015., Pizzi et al., 2016). Emotional processing deficits in patients with TLE lead to poor psychosocial adjustment, reduced quality of life, and high rates of depression and anxiety (Wendling et al., 2013; Cohn et al., 2014; Szaflarski et al., 2014; Bora, \& Meletti, 2016).

In addition, the amygdala, insula, anterior singular cortex, medial prefrontal cortex, and ventricular-lateral region of the prefrontal cortex include key neural substrates and es- sential for emotional processing and regulation (Perez et al., 2016; Mueller et al., 2017). Furthermore, neuroimaging studies have indicated the role of the amygdala and insula in healthy emotional processing (Dellacherie et al., 2011; Schmidt et al., 2014; Mier et al., 2014; Monti, $\&$ Meletti, 2015; Pizzi et al., 2016). The association between increased amygdala reactivity and heightened negative emotions has also been reported in several studies (Bonora et al., 2011; Townsend et al., 2013; Goodman et al., 2014; Gomez-Ibañez, Urrestarazu, \& Viteri, 2014; Gaffrey, Barch, \& Luby, 2016).

Emotional processing is a procedure by which emotional distress is absorbed and reduced; accordingly, one's behavior and experiences continue without disruption. Difficulties in emotional processing could occur at different stages, including recording, evaluation, experiencing, awareness, and emotional expression, leading to behavioral symptoms (Baker, et al., 2010). Besides, healthy emotional processing is essential for emotional and social functioning (Brand et al., 2012; Ho et al., 2014; Marusak, Martin, Etkin, \& Thomason, 2015).

Various studies have revealed deficits in the processing and recognition of facial emotions in patients with TLE (Wendling et al., 2013; Amlerova et al., 2014; Meletti et al., 2014; Monti, \& Meletti, 2015); however, no research has been conducted on the emotional processing styles of these patients. Moreover, some researchers have stated that emotional dysfunction is more prevalent in individuals with epilepsy initiation of before the age of 6 years (early-onset), compared to those with epileptic sei- 
zures onset of after the age of 6 years (late-onset) (Hlobil, Rathore, Alexander, Sarma, \& Radhakrishnan, 2008). The studies have also indicated a relationship between the age of patients and their severity of emotional impairment (Meletti et al., 2003; Brand et al., 2012; Amlerova et al., 2014; Zhao et al., 2014). Nevertheless, most of these studies addressed some areas of emotional dysfunctions, such as facial emotion processing and disregarded other aspects of emotional processing, like emotional processing styles.

Besides, TLE has a chronic nature and impacts the areas involved in emotional recognition and processing due to the damages to the emotional processing circuits; therefore, identifying the pathology of emotional components, like emotional processing in these individuals, compared to healthy groups is one of the strategies to prevent these patients from being exposed to emotional problems. Additionally, preventing the exacerbation of emotional dysfunction could also be effective in providing complementary therapies for TLE patients.

Furthermore, data on emotional processing styles in temporal lobe epilepsy are scarce. However, a great body of literature investigated the dysfunction of emotional processing, like facial emotion processing in TLE patients. Moreover. The present study considered the other aspects of emotional processing, including the emotional processing styles, compared to facial emotion processing, addressed in the previous researches.

Therefore, the emotional processing styles are expected to be more maladaptive in this group, compared to the non-TLE individuals; further dysfunction rates are also expected in patients with an early-onset, compared to those experiencing a late-onset TLE. Accordingly, the primary purpose of this study was to investigate the emotional processing between the TLE and non-epileptic groups; the secondary goal was to explore the extent of emotional processing deficits between the early- and late-onset epilepsy patients.

\section{Methods}

In this cross-sectional study, the samples were selected after receiving a definitive diagnosis of TLE and undergoing Electroencephalography (EEG) by a neurologist. The study participants were chosen from Chamran Hospital (neurology clinic) and the Iranian Epilepsy Association in 2017. Then, according to the inclusion and exclusion criteria, 60 TLE patients, including 30 early-onset and 30 late-onset TLE patients, were recruited using a purposive sampling technique. Besides, 60 healthy subjects from the general population were selected according to the inclu- sion and exclusion criteria by convenience/purposive sampling method.

The inclusion criteria were the age ranged 20-50 years, being educated to at least high school, not being affected by severe mental and personality disorders, not using psychotropic drugs or any other medication with adverse effects on the emotions and mood, as well as no drug and alcohol use or dependency for all participants. Moreover, the specific inclusion criterion for the TLE, the patient group, had TLE diagnosed at or before the age of 6 years (for the early-onset group), as well as having TLE diagnosed after the age of 6 years (for the late-onset group). The exclusion criterion was an unwillingness to participate in the study.

Then, all participants entered the study following a psychiatric interview to exclude patients with severe psychiatric and substance use disorders. Next, they provided signed written informed consent forms. Initially, all TLE patients were compared to 60 healthy individuals regarding emotional processing by Baker's Emotional Processing Scale (Baker, Thomas, Thomas, \& Owens, 2007). Furthermore, the early- and late-onset TLE patients were compared concerning emotional processing. Descriptive statistics (mean and standard deviation) and Multivariate Analysis of Variance (MANOVA) were used to analyze the collected data by SPSS.

To observe the ethical considerations, all procedures of the current study were per the ethical standard of the institutional and national research committee. Some ethical considerations of this study were obtaining informed consent from the subjects; non-disclosure of subjects' personal and identity characteristics; reassuring the subjects about their data confidentially, and qualitatively describing the subjects' test performance data to them, if desired.

The following tools were applied in the present study to obtain the required data:

Emotional Processing Scale (EPS): EPS is a 38-item self-administered scale developed by Baker et al. (Baker, Thomas, Thomas, \& Owens, 2007) to measure emotional processing styles. Each item is scored on a 5-point Likert-type scale (strongly disagree to strongly agree). This scale has three main areas; difficulty in recognizing and experiencing emotions, difficulty in controlling and expressing emotions, and inadequate emotional processing. According to the scale, emotional processing styles are classified into three domains (1- difficulties in emotional recognition and experience, 2- difficulties in emotional control and expression, \& 3-inadequate emotional processing). 
EPS includes 8 subscales (intrusion, suppression, the lack of attunement, the lack of control, dissociation, avoidance, discordant, and externalization). Difficulties in emotion recognition and experience cover the lack of attunement, discordant, and externalization. The domain of difficulties in emotional control and expression includes suppression, dissociation, avoidance, and the lack of control. Inadequate emotional processing comprises intrusion. Cronbach's alpha coefficient and test-retest reliability of this scale were reported as 0.92 and 0.79 , respectively (Baker, Thomas, Thomas, \& Owens, 2007). In a preliminary study on 40 students, Lotfi, Abolghasemi, \& Narimani, (2014) reported -0.54 as the correlation coefficient of EPS with the emotion regulation scale of $\alpha=0.01$. Cronbach's alpha coefficient of this inventory was calculated as 0.95 in another study (Narimani, 2013).

\section{Results}

The relevant results of demographic variables in the epileptic patients suggested that most of the samples were male (66.7\%), single (56.7\%), high school graduate/ dropout (55\%), employed (53.3\%), and of middle socioeconomic status $(76.7 \%)$. In the non-epileptic group, the majority of subjects were male $(53.1 \%), 58.3 \%$ had bachelor's and master's degrees, 57.1\% were married, 60\% were employed, and $70 \%$ were of middle socioeconomic status. These study groups demonstrated no significant differences in the demographic variables.

Table 1 presents the emotional processing scores of the study groups (epileptic and non-epileptic). According to the results, the mean value of emotional processing components in the people with epilepsy was higher than that of the non-epileptics.

MANCOVA was used for data analysis. Prior to the MANOVA test, the Kolmogorov-Smirnov test was used for establishing the normality of the study variables. The non-significance of the test indicated that all studied variables had normal distribution $(\mathrm{P}<0.05)$.

The MANOVA data regarding emotional processing in the epileptic and non-epileptic groups are presented in

Table 1. Mean $\pm S D$ scores of the emotional processing subscales in the patients with TLE and the non-epileptic group

\begin{tabular}{ccc}
\hline & & Mean \pm SD \\
\cline { 2 - 3 } & TLE Group & Non-epileptic Group \\
\hline Intrusion & $17.13 \pm 3.77$ & $13.58 \pm 5.10$ \\
\hline Suppression & $7.08 \pm 3.75$ & $5.39 \pm 3.23$ \\
\hline The lack of attunement & $8.42 \pm 3.51$ & $6.30 \pm 3.34$ \\
\hline The lack of control & $7.38 \pm 4.10$ & $4.91 \pm 3.10$ \\
\hline Dissociation & $7.46 \pm 3.37$ & $5.98 \pm 2.46$ \\
\hline Avoidance & $7.43 \pm 2.91$ & $6.15 \pm 2.70$ \\
\hline Discordant & $16.30 \pm 5.95$ & $9.84 \pm 4.42$ \\
\hline Externalization & $7.43 \pm 2.45$ & $6.08 \pm 2.42$ \\
\hline
\end{tabular}

CLINICAL PSYCH $\oplus$ LOGY

\begin{tabular}{lccccccc}
\hline Effect & Tests & Value & F & Hypothesis df & Error df & P & Partial Eta Squared \\
\hline \multirow{3}{*}{ Group } & Pilla's Trace & 0.327 & 6.75 & 8 & 111 & 0.001 & 0.327 \\
& Wilk's Lambda & 0.673 & 6.75 & 8 & 111 & 0.001 & 0.327 \\
& Hoteling's Trace & 0.487 & 6.75 & 8 & 111 & 0.001 & 0.327 \\
& Roy's Largest Root & 0.487 & 6.75 & 8 & 111 & 0.001 & 0.327 \\
\hline
\end{tabular}


Table 3. The results of between-subject effects of emotional processing subscales in patients with TLE and non-epileptics

\begin{tabular}{|c|c|c|c|c|c|c|c|}
\hline Source & Dependent Variable & $\begin{array}{l}\text { Type III Sum of } \\
\text { Squares }\end{array}$ & df & $\begin{array}{l}\text { Mean } \\
\text { Square }\end{array}$ & $\mathbf{F}$ & $\mathbf{P}$ & $\begin{array}{l}\text { Partial Eta } \\
\text { Squared }\end{array}$ \\
\hline \multirow{8}{*}{ Group } & Intrusion & 379.55 & 1 & 379.55 & 18.81 & 0.000 & 0.138 \\
\hline & Suppression & 85.76 & 1 & 85.76 & 6.96 & 0.009 & 0.056 \\
\hline & The lack of attunement & $134 . .94$ & 1 & $134 . .94$ & 11.46 & 0.001 & 0.089 \\
\hline & The lack of control & 183.34 & 1 & 183.34 & 13.82 & 0.000 & 0.105 \\
\hline & Dissociation & 65.88 & 1 & 65.88 & 7.54 & 0.007 & 0.060 \\
\hline & Avoidance & 49.90 & 1 & 49.90 & 6.31 & 0.013 & 0.051 \\
\hline & Discordant & 1254.09 & 1 & 1254.09 & 45.58 & 0.000 & 0.279 \\
\hline & Externalization & 54.67 & 1 & 54.67 & 9.17 & 0.003 & 0.072 \\
\hline \multirow{8}{*}{ Error } & Intrusion & 2380.31 & 118 & 20.17 & & & \\
\hline & Suppression & 1452.96 & 118 & 12.31 & & & \\
\hline & The lack of attunement & 1388.70 & 118 & 11.76 & & & \\
\hline & The lack of control & 1564.67 & 118 & 13.26 & & & \\
\hline & Discosintion & 102070 & 110 & בד 0 & & & \\
\hline & Avoidance & 932.96 & 118 & 7.90 & & & \\
\hline & Discordant & 3246.07 & 118 & 27.50 & & & \\
\hline & Externalization & 703.31 & 118 & 5.96 & & & \\
\hline
\end{tabular}

Table 4. The MANOVA results for emotional processing subscales between the early- and late-onset TLE groups

\begin{tabular}{cccccccc}
\hline Effect & Tests & Value & F & Hypothesis df & Error df & P & Partial Eta Squared \\
\hline \multirow{3}{*}{ Group } & Pilla's Trace & 0.328 & 3.11 & 8 & 51 & 0.006 & 0.328 \\
& Wilk's Lambda & 0.672 & 3.11 & 8 & 51 & 0.006 & 0.328 \\
& Hoteling's Trace & 0.488 & 3.11 & 8 & 51 & 0.006 & 0.328 \\
& Roy's Largest Root & 0.488 & 3.11 & 8 & 51 & 0.006 & 0.328 \\
\hline
\end{tabular}

Table 2. the obtained $\mathrm{F}$ ratio was significant at the level of $99 \%$. As a result, the groups (epileptic and non-epileptic) were different, at least in terms of one of the dependent variables (emotional processing) (Table 2).

The obtained data on between-group differences in emotional processing subscales are presented in Table 3. The between-subject effects were significant in all components of emotional processing between the study groups $(\mathrm{P}<0.05)$. In other words, emotional processing dysfunction in patients with TLE was more than of the non-epileptic group.
The MANOVA results regarding the emotional processing function between the early- and late-onset TLE patients are presented in Table 4. The obtained $\mathrm{F}$ ratio was significant at the level of $99 \%$. As a result, the groups (epileptic and non-epileptic) were different, at least in terms of one subscale of emotional processing.

According to Table 5, the between-subject effects were significant for three subscales, including intrusion, the lack of attunement, and dissociation between the study groups $(\mathrm{P}<0.05)$. In other words, emotional processing in the subscales of including discordant, the lack of attunement, and 
Table 5. The results of between-subject effects of emotional processing subscales between the early- and late-onset TLE groups

\begin{tabular}{|c|c|c|c|c|c|c|c|}
\hline Source & Dependent variable & $\begin{array}{l}\text { Type III Sum of } \\
\text { Squares }\end{array}$ & df & $\begin{array}{c}\text { Mean } \\
\text { Squared }\end{array}$ & $\mathbf{F}$ & $\mathbf{P}$ & $\begin{array}{l}\text { Partial Eta } \\
\text { Squared }\end{array}$ \\
\hline \multirow{8}{*}{ Group } & Intrusion & 57.38 & 1 & 57.38 & 4.24 & 0.044 & 0.068 \\
\hline & Suppression & 20.82 & 1 & 20.82 & 1.48 & 0.228 & 0.025 \\
\hline & The lack of attunement & 58.51 & 1 & 58.51 & 5.06 & 0.028 & 0.080 \\
\hline & The lack of control & 12.53 & 1 & 12.53 & 0.74 & 0.393 & 0.013 \\
\hline & Dissociation & 113.51 & 1 & 113.51 & 11.79 & 0.001 & 0.169 \\
\hline & Avoidance & 17.48 & 1 & 17.48 & 2.09 & 0.153 & 0.035 \\
\hline & Discordant & 2.15 & 1 & 2.15 & 0.06 & 0.808 & 0.001 \\
\hline & Externalization & 6.66 & 1 & 6.66 & 1.10 & 0.298 & 0.019 \\
\hline \multirow{9}{*}{ Error } & Intrusion & 784.89 & 58 & 13.53 & & & \\
\hline & Suppression & 813.05 & 58 & 14.01 & & & \\
\hline & The lack of attunement & 669.59 & 58 & 11.54 & & & \\
\hline & The lack of control & 981.55 & 58 & 16.92 & & & \\
\hline & & & & & & & \\
\hline & Dissociation & 558.19 & 58 & 9.62 & & & \\
\hline & Avoidance & 483.83 & 58 & 8.34 & & & \\
\hline & Discordant & 2089.35 & 58 & 36.02 & & & \\
\hline & Externalization & 350.06 & 58 & 6.03 & & & \\
\hline
\end{tabular}

dissociation were higher in patients with early-onset TLE, than the late-onset group.

\section{Discussion}

This study aimed to identify the emotional processing function in patients with TLE. The present study results supported the hypothesis concerning the maladaptive emotional processing styles components between TLE patients versus healthy individuals. The research findings suggested the inappropriate emotional processing styles, including intrusion, suppression, the lack of attunement, the lack of control, dissociation, avoidance, discordant, and externalized, were more common in patients with TLE. The larger eta effect size belonged to the discordant (difficulties in emotional recognition and experience), intrusion (inadequate emotional processing), and the lack of control (difficulties in emotional control and expression). Therefore, the emotional processing was impaired in all 3 domains of emotional processing styles (difficulties in emotional recognition and experience, difficulties in emotional control and expression, \& inadequate emotional processing), compared to the non-epileptics. Based on the quantity of, the effect size, the more impairment was respectively observed in difficulties in emotional recognition and experience, inadequate emotional processing, and difficulties in emotional control and expression.

A study investigated emotion-processing styles in patients with Psychogenic Non-Epileptic Seizures (PNES); the relevant data suggested more deficiencies of emotion processing across all dimensions of emotional processing styles in patients with PNES, compared to healthy individuals (Novakova, Howlett, Baker, \& Reuber, 2015). Furthermore, various studies reported the deficiency of facial emotion processing in TLE patients and suggested further difficulties in emotion recognition in these patients, compared to the non-epileptics; these findings were in line with those of the present study (Wendling et al., 2013; Amlerova et al., 2014; Meletti et al., 2014; Monti, \& Meletti, 2015). However, scholars overlooked exploring emotional processing styles in TLE patients. 
Bartolomei et al. (2005) stated that severe emotional changes in epileptic patients are associated with decreased concurrency between signals recorded from neural networks involved in emotional processing; such alternations could especially be detected between the orbital-frontal cortex and the amygdala. Impairments in functional communication cause disruption in emotion regulation and altered behaviors during seizures, such as severe restlessness, anger expression, and fear in patients.

The second study hypothesis about more frequent dysfunctions in emotional processing in early-onset TLE patients, compared to the late-onset TLE group was also supported. The emotional processing was compared in the early- and late-onset groups in terms of the three components, including dissociation (emotional separation), the lack of attunement (attitude towards the ineffectiveness of the emotions or them being meaninglessness) and intrusion (continuous, repetitive and disturbing negative emotional experiences, and the slow stifling or suppression of negative emotions); accordingly, those with early-onset epilepsy used these three emotional strategies more often, compared to the late-onset group. Moreover, the eta effect size was more significant for dissociation, the lack of attunement, and intrusion, respectively, in them. Besides, the early-onset of epilepsy (i.e. $\leq 6$ years) is associated with poorer emotional processing as well as impaired ability in facial emotion recognition. Studies have reported such defects in children with TLE (Brand et al., 2012).

Meletti et al. (2003) studied the ability to recognize facial emotion processing in Drug-Resistant Focal Epilepsy (DRFE) patients. They concluded that seizures with fever and early-onset epilepsy are strongly linked to the severity of emotion recognition deficits.

Some studies have also indicated that early-onset epilepsy and its location (the right hemisphere or in both hemispheres) results in more deficits in processing emotions, such as fear, sadness, and disgust than anger and happiness (Tanaka et al., 2013).

Another study assessed facial emotion recognition in 140 patients with chronic TLE for 5 years; they reported extensive defects in recognizing negative emotions in the studied samples. Specifically, those with early-onset epileptic seizures that occurred in the right hemisphere or both hemispheres had more severe emotional deficits (Bonora et al., 2011).

Additionally, Amlerova et al. (2014) argued that emotion recognition and social cognition in individuals with TLE are influenced by some characteristics, including early- onset epilepsy, longer duration of illness, and a history of damage and brain injury in early childhood. Therefore, the present study findings are consistent with those of the previous studies.

A limitation of this study was using self-administrated questionnaires in assessing emotional processing. These questionnaires may be limited, particularly in identifying emotional processing, and may not be as accurate as objective instruments on how emotions are processed. Moreover, to obtain social approval, the study participants may have had a positive bias. Using more objective measurements is suggested for future studies. In addition, further research could measure patients' other emotional functions concerning their social context and level of emotional, social adjustment through interviews and specialized checklists. Besides, in previous studies, no specific criteria were considered for early- or late-onset TLE, and we also used the age of 6 years considered by previous studies; accordingly, in future studies, this issue needs to be regarded more closely and based on further evidence on the pathophysiology of the early- or late-onset TLE diseases.

Regarding recognition and understanding, emotional dysfunctions, such as emotional processing styles, could be useful for the complementary treatment and prevention of exacerbation of emotional dysfunction. Furthermore, neurologists could refer to psychotherapists for additional psychological interventions that provide some skills to overcome the interpersonal and social communication deficiency induced by emotional processing dysfunctions.

\section{Conclusion}

The obtained results suggested that emotional processing in the TLE patients was more maladaptive, compared to the non-epileptics. Moreover, the study findings revealed that early-onset TLE patients had more dysfunctions in emotional processing, compared to the late-onset TLE individuals.

\section{Ethical Considerations}

\section{Compliance with ethical guidelines}

All ethical principles were considered in this article. The participants were informed about the purpose of the research and its implementation stages; they were also assured about the confidentiality of their information; Moreover, They were allowed to leave the study whenever they wish, and if desired, the results of the research would be available to them. 


\section{Funding}

This research did not receive any specific grant from funding agencies in the public, commercial, or not-forprofit sectors.

\section{Authors' contributions}

All authors contributed equally in preparing all parts of the research.

\section{Conflict of interest}

The authors declared no conflicts of interest.

\section{Acknowledgments}

The authors wish to acknowledge their gratitude to all the staff and neurologists of Chamran Hospital, as well as the Epilepsy Society of Iran, for their valuable assistance to conduct this study.

\section{References}

Amlerova, J., Cavanna, A. E., Bradac, O., Javurkova, A., Raudenska, J., \& Marusic, P. (2014). Emotion recognition and social cognition in temporal lobe epilepsy and the effect of epilepsy surgery. Epilepsy \& Behavior, 36, 86-89. [DOI:10.1016/j. yebeh.2014.05.001] [PMID]

Baker, R., Thomas, S., Thomas, P. W., \& Owens, M. (2007). Development of an emotional processing scale. Journal of Psychosomatic Research, 62(2), 167-78. [DOI:10.1016/i.jpsychores.2006.09.005] [PMID]

Baker, R., Thomas, S., Thomas, P. W., Gower, P., \& Santonastaso, M., et al. (2010). The Emotional Processing Scale: Scale refinement and abridgement (EPS-25). Journal of Psychosomatic Research, 68(1), 83-88. [DOI:10.1016/i.jpsychores.2009.07.007] [PMID]

Bartolomei, F., Trébuchon, A., Gavaret, M., Régis, J., Wendling, F., \& Chauvel, P. (2005). Acute alteration of emotional behaviour in epileptic seizures is related to transient desynchrony in emotion-regulation networks. Clinical Neurophysiology, 116(10), 2473-9. [DOI:10.1016/j.clinph.2005.05.013] [PMID]

Baulac, M., De Boer, H., Elger, C., Glynn, M., Kälviäinen, R., Little, A., Mifsud, J., Prucca, E., Pitkänen, A., Ryvlin, P. (2015). Epilepsy priorities in Europe: A report of the ILAE-IBE epilepsy advocacy Europe task force. Epilepsia, 56(11), 1687-95. [DOI:10.1111/epi.13201] [PMID] [PMCID]

Bonora, A., Benuzzi, F., Monti, G., Mirandola, L., Pugnaghi, M., Nichelli, P., \& Meletti, S. (2011). Recognition of emotions from faces and voices in medial temporal lobe epilepsy. Epilepsy $\mathcal{E}$ Behavior, 20(4), 648-54. [DOI:10.1016/i.yebeh.2011.01.027] [PMID]
Bora, E., \& Meletti, S. (2016). Social cognition in temporal lobe epilepsy: a systematic review and meta-analysis. Epilepsy $\mathcal{E}$ Behavior, 60, 50-7. [DOI:10.1016/j.yebeh.2016.04.024] [PMID]

Brand, J. G., Mindt, M. R., Schaffer, S. G., Alper, K. R., Devinsky, O., \& Barr, W. B. (2012). Emotion processing bias and age of seizure onset among epilepsy patients with depressive symptoms. Epilepsy \& Behavior, 25(4), 552-557. [DOI:10.1016/j. yebeh.2012.09.031] [PMID]

Cohn, M., St-Laurent, M., Barnett, A., \& McAndrews, M. P. (2014). Social inference deficits in temporal lobe epilepsy and lobectomy: Risk factors and neural substrates. Social Cognitive and Affective Neuroscience, 10(5), 636-44. [DOI:10.1093/scan/ nsu101] [PMID] [PMCID]

Cukiert, A., Cukiert, C. M., Burattini, J. A., \& Lima, A. M. (2014) Seizure outcome after hippocampal deep brain stimulation in a prospective cohort of patients with refractory temporal lobe epilepsy. Seizure, 23(1), 6-9. [DOI:10.1016/j.seizure.2013.08.005] [PMID]

Dellacherie, D., Hasboun, D., Baulac, M., Belin, P., \& Samson, S. (2011). Impaired recognition of fear in voices and reduced anxiety after unilateral temporal lobe resection. Neuropsychologia, 49(4), 618-29. [DOI:10.1016/j.neuropsychologia.2010.11.008] [PMID]

Elliott, J. O., \& Richardson, V. E. (2014). The biopsychosocial model and quality of life in persons with active epilepsy. Epilepsy \& Behavior, 41, 55-65. [DOI:10.1016/j.yebeh.2014.09.035] [PMID]

Gaffrey, M. S., Barch, D. M., \& Luby, J. L. (2016). Amygdala reactivity to sad faces in preschool children: An early neural marker of persistent negative affect. Developmental Cognitive Neuroscience, 17, 94-100. [DOI:10.1016/i.dcn.2015.12.015] [PMID] [PMCID]

Gomez-Ibañez, A., Urrestarazu, E., \& Viteri, C. (2014). Recognition of facial emotions and identity in patients with mesial temporal lobe and idiopathic generalized epilepsy: An eye-tracking study. Seizure, 23(10), 892-8. [DOI:10.1016/j.seizure.2014.08.012] [PMID]

Goodman, M., Carpenter, D., Tang, C. Y., Goldstein, K. E., Avedon, J., Fernandez, N., et al. (2014). Dialectical behavior therapy alters emotion regulation and amygdala activity in patients with borderline personality disorder. Journal of Psychiatric research, 57, 108-16. [DOI:10.1016/i.jpsychires.2014.06.020 [PMID] [PMCID]

Hlobil, U., Rathore, C., Alexander, A., Sarma, S., \& Radhakrishnan, K. (2008). Impaired facial emotion recognition in patients with mesial temporal lobe epilepsy associated with hippocampal sclerosis (MTLE-HS): Side and age at onset matters. Epilepsy Research, 80(2-3), 150-7. [DOI:10.1016/i.eplepsyres.2008.03.018] [PMID]

Ho, T. C., Yang, G., Wu, J., Cassey, P., Brown, S. D., Hoang, N., Chesney, M. A. (2014). Functional connectivity of negative emotional processing in adolescent depression. Journal of Affective Disorders, 155, 65-74. [DOI:10.1016/j.jad.2013.10.025] [PMID] [PMCID]

Kang, J. Y., Wu, C., Tracy, J., Lorenzo, M., Evans, J., Nei, M., Skidmore, C., Mintzer, S., Sharan, A. D., Sperling, M. R. (2016). Laser interstitial thermal therapy for medically intractable mesial temporal lobe epilepsy. Epilepsia, 57(2), 325-34. [DOI:10.1111/epi.13284] [PMID] 
Lotfi, S., Abolghasemi, A., \& Narimani, M. (2014). A comparison of emotional processing and fear of positive/negative evaluations in women with social phobia and normal women. JSR, 14(53), 101-11.

Lv, R. J., Sun, Z. R., Cui, T., Guan, H. Z., Ren, H. T., \& Shao, $X$. Q. (2014). Temporal lobe epilepsy with amygdala enlargement: A subtype of temporal lobe epilepsy. BMC Neurology, 14(1):194-202. [DOI:10.1186/s12883-014-0194-z] [PMID] [PMCID]

Marusak, H. A., Martin, K. R., Etkin, A., \& Thomason, M. E. (2015). Childhood trauma exposure disrupts the automatic regulation of emotional processing. Neuropsychopharmacology, 40(5), 1250-8. [DOI:10.1038/npp.2014.311] [PMID] [PMCID]

Meletti, S., Benuzzi, F., Rubboli, G., Cantalupo, G., Maserati, M. S., Nichelli, P., \& Tassinari, C. (2003). Impaired facial emotion recognition in early-onset right mesial temporal lobe epilepsy. Neurology, 60(3), 426-31. [DOI:10.1212/WNL.60.3.426] [PMID]

Meletti, S., Cantalupo, G., Santoro, F., Benuzzi, F., Marliani, A. F., Tassinari, C. A., \& Rubboli, G. (2014). Temporal lobe epilepsy and emotion recognition without amygdala: A case study of Urbach Wiethe disease and review of the literature. Epileptic Disorders, 16(4), 518-27. [DOI:10.1684/epd.2014.0696] [PMID]

Mier, D., Lis, S., Zygrodnik, K., Sauer, C., Ulferts, J., Gallhofer, B., \& Kirsch, P. (2014). Evidence for altered amygdala activation in schizophrenia in an adaptive emotion recognition task. Psychiatry Research: Neuroimaging, 221(3), 195-203. [DOI:10.1016/j.pscychresns.2013.12.001] [PMID]

Monti, G., \& Meletti, S. (2015). Emotion recognition in temporal lobe epilepsy: A systematic review. Neuroscience $\mathcal{E}$ Biobehavioral Reviews, 55, 280-93. [DOI:10.1016/j.neubiorev.2015.05.009] [PMID]

Mueller, F., Lenz, C., Dolder, P. C., Harder, S., Schmid, Y., Lang, U. E., Liechti, M. E., \& Borgwardt, S. (2017). Acute effects of LSD on amygdala activity during processing of fearful stimuli in healthy subjects. Translational Psychiatry, 7(4), e1084. [DOI:10.1038/tp.2017.54] [PMID] [PMCID]

Narimani, M. (2013). The effectiveness of impulse control training on emotional processing, impulsivity and distractibility of students with dyscalculia. Journal of Learning Disability, 2(2), 101-22. [DOI:10.5812/ijhrba.10791] [PMID] [PMCID]

Novakova, B., Howlett, S., Baker, R., \& Reuber, M. (2015). Emotion processing and psychogenic non-epileptic seizures: A cross-sectional comparison of patients and healthy controls. Seizure, 29, 4-10. [DOI:10.1016/j.seizure.2015.03.007] [PMID]

Perez, D. L., Vago, D. R., Pan, H., Root, J., Tuescher, O., Fuchs, et al. (2016). Frontolimbic neural circuit changes in emotional processing and inhibitory control associated with clinical improvement following transference-focused psychotherapy in borderline personality disorder. Psychiatry and clinical neurosciences, 70(1), 51-61. [DOI:10.1111/pcn.12357] [PMID] [PMCID]

Pizzi, S. D., Chiacchiaretta, P., Mantini, D., Bubbico, G., Ferretti, A., Edden, R. A., et al. (2017). Functional and neurochemical interactions within the amygdala-medial prefrontal cortex circuit and their relevance to emotional processing. Brain Structure and Function, 222(3), 1267-79. [DOI:10.1007/s00429016-1276-z] [MID] [MCID]

Schmidt, A., Borgwardt, S., Gerber, H., Wiesbeck, G. A., Schmid, O., Riecher-Rössler, et al. (2014). Acute effects of heroin on negative emotional processing: Relation of amygdala activity and stress-related responses. Biological Psychiatry, 76(4), 289296. [DOI:10.1016/j.biopsych.2013.10.019] [MID]

Szaflarski, J. P., Allendorfer, J. B., Heyse, H., Mendoza, L., Szaflarski, B. A., \& Cohen, N. (2014). Functional MRI of facial emotion processing in left temporal lobe epilepsy. Epilepsy $\mathcal{E}$ Behavior, 32, 92-9. [DOI:10.1016/i.yebeh.2014.01.012] [PMID]

Tanaka, A., Akamatsu, N., Yamano, M., Nakagawa, M., Kawamura, M., \& Tsuji, S. (2013). A more realistic approach, using dynamic stimuli, to test facial emotion recognition impairment in temporal lobe epilepsy. Epilepsy \& Behavior, 28(1), 126. [DOI:10.1016/i.yebeh.2013.03.022] [PMID]

Townsend, J. D., Torrisi, S. J., Lieberman, M. D., Sugar, C. A., Bookheimer, S. Y., \& Altshuler, L. L. (2013). Frontal-amygdala connectivity alterations during emotion downregulation in bipolar I disorder. Biological psychiatry, 73(2), 127-35. [DOI:10.1016/j.biopsych.2012.06.030] [PMID] [PMCID]

Wendling, A. S., Hirsch, E., Wisniewski, I., Davanture, C., Ofer, I., Zentner, J., et al. (2013). Selective amygdalohippocampectomy versus standard temporal lobectomy in patients with mesial temporal lobe epilepsy and unilateral hippocampal sclerosis. Epilepsy Research, 104(1-2), 94-104. [DOI:10.1016/i. eplepsyres.2012.09.007] [PMID]

Yilmazer-Hanke, D., O’Loughlin, E., \& McDermott, K. (2016). Contribution of amygdala pathology to comorbid emotional disturbances in temporal lobe epilepsy. Journal of Neuroscience Research, 94(6), 486-503. [DOI:10.1002/jnr.23689] [PMID]

Zhao, F., Kang, H., You, L., Rastogi, P., Venkatesh, D., \& Chandra, M. (2014). Neuropsychological deficits in temporal lobe epilepsy: A comprehensive review. Annals of Indian Academy of Neurology, 17(4), 374. [DOI:10.4103/0972-2327.144003] [PMID] [PMCID] 
This Page Intentionally Left Blank 